of primary MSC characteristics, these MSC lines are expected to be valuable tools for the research on Regenerative Medicine for OA as part of Tissue Engineering in vitro models.

Acknowledgement: Xunta de Galicia and European Union (European Social Fund-ESF); Deputación da Coruña; Rede de Investigación en Células Nai e Terapia Celular (R2016/036, R2014/050 and CN2012/142) and Grupos con Potencial de Crecemento (GPC2014/048) (Xunta de Galicia); CIBER-BNN; Fundación Española de Reumatología (FER); Fondo de Investigación en Salud (FIS) (PI17/ 02197).

Disclosure of Interests: María Piñeiro-Ramil: None declared, Rocío CastroViñuelas: None declared, Clara Sanjurjo-Rodríguez: None declared, Silvia Rodríguez-Fernández: None declared, Tamara Hermida Gómez: None declared, Francisco Javier de-Toro-Santos: None declared, Francisco J. Blanco Consultant for: AbbVie, Bioiberica, BMS, GSK, Grünenthal, Janssen, Lilly, Pfizer, Regeneron, Roche, Sanofi, TRB Chemedica, and UCB, Isaac Fuentes-Boquete: None declared, SILVIA MARIA DÍAZ-PRADO: None declared DOI: 10.1136/annrheumdis-2019-eular.6717

\section{AB0103 A NOVEL PROTECTIVE ROLE OF GPNMB/ OSTEOACTIVIN IN POST-TRAUMATIC OSTEOARTHRITIS}

Asaad Al Adlaan ${ }^{1}$, Nazar Hussein ${ }^{1}$, Fatima Jaber ${ }^{1}$, Fayez Safadi ${ }^{1,2} .{ }^{1}$ Rootstown, Anatomy and Neurobiology, Musculoskeletal Research Group, Rootstown, United States of America; ${ }^{2}$ Akron Children's Hospital, Rebecca D Considine Research Institute, Akron Children's Hospital, Akron, United States of America

Background: Osteoarthritis $(\mathrm{OA})$ is a chronic joint disease causes irreversible damage to the articular cartilage resulting in loss of joint function and subchondral bone remodeling. There is no cure for OA and currently the available treatment like pain management, and joint replacement surgery is used to treat this disease. GPNMB/Osteoactivin is a type-I transmembrane protein plays a vital role in osteogenesis and bone remodeling,

Objectives: Howver, the function of GPNMB/Osteoactivin in chondrogenesis and cartilage repair is unknown. Hence, in this study, we examined the role of GPNMB/Osteoactivin using post-traumatic osteoarthritis model and ex vivo chondrocytes cell culture and assessing the effects of GPNMB/Osteoactivin as antiinflammatory factor in chondrocytes.

Methods: We first examined the expression GPNMB/Osteoactivin in noramal cartilage. Next, we assessed the effects of recombinant GPNMB/Osteoactivin on mouse primary chondrocytes profeliation/viabiltiy and extracellular matrix marekrs.

Results: We founad that GPNMB/Osteoactivin treatment did not have any effect on cell prolfieration/viability, howwever, there was an increased in Colllagen Type II and aggrecan expression in a dose- and time-dependent manner. These data sugges that GPNMB/Osteoactivin palys a role in cartilage maintainance. Next we determined whether GPNMB/Osteoactivin contributes to the OA disease progression. Human osteoarthritic damaged and undamaged cartilage harvested following knee replacement were used to examine GPNMB/Osteoactivin exmression and founad that GPNMB/Osteoactivin mRNA was 6-7-fold increased in damaged compared to undamaged cartilage. Similar results were obtained from mouse primary chondrocytes treated with IL1-b and showed a significant increase (4-fold) in GPNMB/Osteoactivin mRNA compared to untreated control. Next, we evalauted the effects of GPNMB/Osteoactivin deficiency on the OA progression usign the DMM model. GPNMB/Osteoactivin muntat (DBA/2J) mice showed significant increased in OARSI scores compared to WT mice.

Conclusion: Taken together, these data are the first to report a role for GPNMB/ Osteoactivin in cartilage maintanance and protection again the prgoression of $O A$.

\section{REFERENCES}

[1] The surgical destabilization of the medial meniscus (DMM) model of osteoarthritis in the 129/SvEv mouse. Methods in Molecular Biology: Osteoporosis and Osteoarthritis. Loeser RF, Goldring SR, Scanzello CR, Goldring MB. Osteoarthritis: A disease of the joint as an organ. Arthritis and Rheumatism. 2012. pp. 1697-1707.

[2] Zhou, L., Zhuo, H., Ouyang, H., Liu, Y., Yuan, F., Sun, L.,. .. \& Liu, H. (2017). Glycoprotein non-metastatic melanoma protein b (Gpnmb) is highly expressed in macrophages of acute injured kidney and promotes M2 macrophages polarization. Cellular immunology, 316, 53-60.

[3] Ripoll, V.M., et al., Gpnmb is induced in macrophages by IFN-gamma and lipopolysaccharide and acts as a feedback regulator of proinflammatory responses. J Immunol, 2007. 178(10): p. 655766.

[4] Karlsson C, Dehne T, Lindahl A, Brittberg M, Pruss A, Sittinger M, Ringe J. Genome-wide expression profiling reveals new candidate genes associated with osteoarthritis. Osteoarthritis and Cartilage. 2010 Apr 1;18(4):581-92.

Acknowledgement: This research was funded in part by the Cook Research Fund, Summa Heatlh System and Ohio Department of Education
Disclosure of Interests: None declared

DOI: 10.1136/annrheumdis-2019-eular.8175

\section{AB0104 $\quad$ ARE POLLUTANTS A NEW RISK FACTOR FOR OSTEOARTHRITIS? RESULTS FROM A SYSTEMATIC LITERATURE REVIEW}

Camille Deprouw ${ }^{1}$, Alice Courties ${ }^{1}$, Jean-Baptiste Fini ${ }^{2}$, Marie-Stéphanie ClergetFroidevaux $^{2}$, Barbara Demeneix ${ }^{2}$, Francis Berenbaum ${ }^{1}$, Jeremie Sellam ${ }^{1}$, Karine Louati ${ }^{1}{ }^{1}$ St Antoine Hospital, Paris, France; ${ }^{2}$ Museum National d'Histoire Naturelle, Paris, France

Background: Prevalence of knee osteoarthritis $(\mathrm{OA})$ has doubled since the mid20th century. Interestingly, this increase cannot be explained solely by longer life expectancy and the obesity epidemics (1). Therefore, other environmental factors known to have increased in the recent years could be part of the explanation.

Objectives: We performed a systematic literature review (SLR) to summarize the existing knowledge about associations between $\mathrm{OA}$ and pollutants.

Methods: Pubmed database was used to identify studies reporting data on OA and pollutants in humans (examples of MeSH terms: "Polychlorinated Biphenyls (PCB)" or "Lead"). Abstracts from international congresses were reviewed for the past 2 years, combined with manual curation. Studies were classified in epidemiological clinical studies, pollutants assessments in ex vivo OA joint tissues, and in vitro effect on human chondrocyte.

Results: As of January 15, 2019, 193 potentially relevant articles were screened. Among them, 14 were selected. After manual curation, a total of 21 full text articles were analyzed. Ten articles reported epidemiological clinical studies. Four articles underlined the link between PCB and OA: in the 3 articles reporting association with past exposure to $\mathrm{PCB}$, the most robust one showed that risk of OA was significantly increased, at least in men (OR 4.1 [95\% Cl: 1.8-11.2] for men and 1.3 [95\% $\mathrm{Cl}$ : 0.8-2.3] for women). In the $4^{\text {th }}$ study, the highest serum concentration of PCB was associated with a higher risk of arthritis: maximal adjusted $\mathrm{OR}(\mathrm{aOR})$ was 3.2 [95\%Cl: 1.6-6.7], $\mathrm{p}<0.01$. However, sensitivity analysis of OA patients did not find any significant association in this study. Two articles focused on serum levels of perfluorooctanoate (PFOA) and perfluorooctane sulfonate (PFOS): aOR to have $\mathrm{OA}$ in the PFOA highest serum level quartile was 1.42 [95\% $\mathrm{Cl}: 1.26-1.59]$, $\mathrm{p}=0.00001$ and 1.55 [95\%Cl: 0.99-2.43]. Data were conflicting for PFOS: aOR to have OA in the PFOS highest serum level quartile was 1.77 [95\% $\mathrm{Cl}: 1.05-2.96]$, $p<0.05$ and 0.76 [95\% $\mathrm{Cl}: 0.68-0.85], p=0.00001$. Two articles showed an association between serum lead levels and knee $O A$ in the general population. In Korea, the risk of radiographic $O A$ was increased in the highest quartile of lead serum level compared to the lowest one (aOR 1.90 [95\% Cl: 1.09-3.32] for men and 1.81 [95\%Cl: 1.17-2.77] for women). In the United States, risk knee radiographic OA correlates with increased lead serum levels. In the last study, household air pollution induced by the cooking heat source was examined: compared to electricity, the risk of OA was increased with the use of liquids (kerosene/paraffin: aOR 1.73 [95\% Cl 1.33-2.25]) or solid (coal, wood: aOR 1.73 [1.32-2.27]), (agriculture/crop: aOR 2.0 [1.47-2.72]). Lead and zinc accumulation was reported in 7 articles: 4 of them analyzed the concentration in $\mathrm{OA}$ joint obtained during arthroplasty and showed highest concentration in cartilage versus bone. In another, lead serum levels were associated with biomarkers of joint tissue metabolism. Two studies with X-ray fluorescence on cadavers found a high accumulation of lead and zinc in the articular cartilage tidemark. Four studies assessed the in vitro effect of pollutants on human chondrocytes: the viability of chondrocytes was reduced in presence of PCB, gold, silver, fluvic acid and bisphenol A.

Conclusion: This SLR suggests a possible link between OA and pollutants but the designs of the studies, the populations investigated and the type of pollutants were still too heterogeneous and limited to conclude definitively that pollutants represent a new environmental risk factor for OA. However, this SLR highlights a critical need for novel epidemiological, clinical and basic research studies in order to identify other potential environmental factors in OA.

\section{REFERENCES}

[1] Wallace, PNAS 114(35), 2017

Disclosure of Interests: None declared

DOI: 10.1136/annrheumdis-2019-eular.5864

\section{AB0105 THE EFFECT OF TROMBOSIT RICH PLASMA APPLICATION ON DEGENERATED CARTILAGE: IN VITRO EXPERIMENTAL STUDY}

Kevser Arapoğlu, Ömer Faruk Șendur, Serhan Sakarya. ADU, PRandM, IZMIR, Turkey

Background: Osteoarthritis is the most common problem among musculoskeletal system disorders. Platelet-rich plasma (PRP) has been suggested to be 
beneficial in the treatment of degenerative musculoskeletal problems. The purpose of this study is to evaluate PRP treatment efficacy on degenerated cartilage. Objectives: In this study, we aimed to determine the efficacy of platelet-rich plasma (PRP) on mechanically damaged chondrocyte cells by using different dose, different duration of exposure and different methods of activation of platelet. Methods: Human source chondrocytes (CHON-001 ATTC CRL-2846) were used in thestudy. Chondrocyte cells were produced in appropriate medium and an experimental cartilage model was created. Theplatelet-rich plasma was produced from platelets obtained by apheresis in the laboratory, from blood of volunteer. Theplatelet-rich plasma was adjusted at five different doses as $4,8 \times 10^{6}, 2,4 \times 10^{6}$ $1.2 \times 10^{6}, 6 \times 10^{5}, 3 \times 10^{5}$. The first group of platelet rich plasma was left intact, the second group was detonated within seven minutes by applying ultrasound waves in water, the third group was activated with calcium chloride and thefourth group was determined as control group. Using a ten microliter pipette tip, a linear damage to theopposite side was created at the widest part of the well. Cell migration was monitored at $0-4-8-24$ and 48 hours at $\times 10$ magnification by in vitro microscopy and wound healing was evaluated by photographing. Migration intervals were determined quantitatively using the program named Image $\mathrm{J}$.

Results: When the rates of recovery were compared to the groups, no significant improvement was observed in the intact and detonated platelet groups at 4-8 and 24 hours compared to the control group. In the third group which was activated with calcium, no significant improvement was observed in all doses at 4 and 8 hours compared to the control group. However, at the 48th hours there was a significant improvement in the doses of $1.2 \times 10^{6}, 2.4 \times 10^{6}$ and $4.8 \times 10^{6}$ compared to the control group $(p<0.0001)$.

There were significant differences in intact and detonated platelets at $3 \times 10^{5}$ and $6 \times 10^{5}$ doses at 48 th hours compared to controlgroup $(p<0.0001)$. Significant improvement was observed in all groups at levels of $1.2 \times 10^{6}$ and a bove ( $p$ $<0.0001)$.

When evaluated in terms of activation, there was a significant improvement in the exploded and intact groups at the 48th hour, compared to the calcium-activated group at doses of $3 \times 10^{5}$ and $6 \times 10^{5}(p<0.01)$.

Conclusion: Cartilage damage is the main pathology in the pathogenesis of osteoarthritis. All doses of PRP used in the study contributed to improvement. Meanwhile, the most critical parameter for platelet migration was timing and significant improvement was started after 48 hours.

\section{REFERENCES}

[1] Nguyen RT, Borg-Stein J, Mclnnis K. Applications of platelet-rich plasma in musculoskeletal and sports medicine: an evidence-based approach. PM R 2011;3(3):226-50.

[2] Filardo G, Kon E, DiMatteo B, Merli ML, Cenacchi A, Fornasari PM, et al. Platelet-rich plasma vs hyaluronicacid to treat knee degenerative pathology: study designand preliminary results of a randomizedcontrolledtrial. BMC MusculoskeletDisord2012;13:229.

[3] Spaková T, Rosocha J, Lacko M, Harvanova D, Gharaibeh A. Treatment of knee joint osteoarthritis with autologous platelet-rich plasma in comparison with hyaluronic acid. Am J Phys Med Rehabil 2012;91(5):411-7.

[4] Virchenko $O$, Aspenberg $P$. How can one platelet injection after tendon injury lead to a stronger tendon after 4 weeks? Interplay between early regeneration and mechanical stimulation. Acta Orthop 2006;77(5):806-12.

Disclosure of Interests: None declared

DOI: 10.1136/annrheumdis-2019-eular.611

\section{AB0106 CHANGES IN THE MIRNA PROFILE AND HYPOXIC BEHAVIOUR OF HUMAN CHONDROCYTES BY THERAPEUTIC NUCLEAR MAGNETIC RESONANCE THERAPY (NMRT)}

Birgit Lohberger ${ }^{1}$, Lukas Weigl ${ }^{2}$, Nicole Stuend ${ }^{1}$, Anda Mann ${ }^{2}$, Andreas Leithner $^{1}$, Werner Kullich ${ }^{3}$, Bibiane Steinecker-Frohnwieser ${ }^{4}$. ${ }^{1}$ Medical University of Graz, Department of Orthopaedics and Trauma, Graz, Austria; ${ }^{2}$ Medical University of Vienna, Division of Special Anaesthesia and Pain Medicine, Vienna, Austria; ${ }^{3}$ Ludwig Boltzmann Institute for Arthritis and Rehabilitation, Saalfelden, Austria; ${ }^{4}$ Ludwig Boltzmann Institute for Arthritis and Rehabilitation, Groebming, Austria

Background: Therapeutically applied nuclear magnetic resonance (NMRT) is discussed to participate in repair processes regarding cartilage and influences pain signaling. Studies concerning NMR therapy implemented within the treatment of patients with degenerative rheumatic diseases outlined pain reduction as the main clinical outcome (1). NMRT is also known to lead to improvements in pain from patients with knee OA due to a chondroprotective effect on the articular cartilage. In spite of this significant reduction in pain, the mechanism of action of NMRT at the cellular level remains to be elucidated.

Objectives: To substantiate the application of NMRT the aim of this work targets the underlying mechanisms at the cellular level. We investigated NMRT induced changes of the miRNA profile of human healthy and OA chondrocytes and studied the respective miRNA targets. Based on the fact that articular cartilage functions as an avascular and hypoxic connective tissue we were further able to demonstrate that NMRT modulation seems to be more pronounced under hypoxic conditions.

Methods: Human primary chondrocytes and the chondrocyte cell line Tc28/2a were used for the experiments.

RNA was extracted using RNEasy Mini Kit and was used as input for the Thermo Fisher Ion Total RNA-Seq Kit v2. Sequencing was performed on lon Proton sequencer using the lon $\mathrm{PI} H \mathrm{H}-\mathrm{Q}^{\mathrm{TM}}$ Sequencing 200 system. Signal processing and base calling was performed using Torrent Suite version 5.6. Hypoxic conditions were established and enabled cell growth in presence of $1-5 \% \mathrm{O} 2$. Expression of miRNAs and target proteins was studied by a standard PCR procedure as well as protein detection by western blot. HDAC activity was measured by HDAC Glo I/II assay.

Results: Characterization of the miRNA profile showed a slight up regulation of miR-24-1-5p and miR-502-5p while miR-25-5p and miR-365a-5p was down regulated. For miR-365a-5p known to directly targeting HDAC and NFkB a decrease of HDAC activity by NMRT was detected. The miR-25-5p target COX2 was changed in expression by NMRT whereas no influence on CDK4 knowing to be controlled by miR-24-1-5p was detected. NMRT treatment of chondrocytes under hypoxic conditions $\left(0-5 \% \mathrm{O}_{2}\right)$ changed the expression profile with respect to NOS, IGF2, PDGF and IGFBP and a change in the expression of Hif1/2 under the influence of IL1b was observed. The hypoxic conditions changed apoptotic behavior of the cells, NMRT showed no influence.

Conclusion: A closer look into the mechanism of the NMRT at the cellular leve revealed a modulatory effect on miRNA, their regulatory units and chondrocytes under hypoxic conditions. The results underline our former results indicating that NMRT counteracts IL-1b induced changes which mean that pain reduction by NMRT might be due to NMRT holding against inflammatory mechanisms under $\mathrm{OA}$

\section{REFERENCES}

[1] Kullich W, Overbeck J, Spiegel HU; Journal of Back and Musculoskeletal Rehabilitation 2013, 26:93-104.

[2] Steinecker-Frohnwieser B, Kullich W, Mann A, Kress H, Weigl L; Exp. Clin. Rheum. 2018, 36, 2:0294-0301.

Disclosure of Interests: Birgit Lohberger: None declared, Lukas Weigl: None declared, Nicole Stuendl: None declared, Anda Mann: None declared, Andreas Leithner: None declared, Werner Kullich Grant/research support from: minor research support for chemicals, Bibiane Steinecker-Frohnwieser Grant/research support from: research support

DOI: 10.1136/annrheumdis-2019-eular.7855

\section{$\mathrm{AB} 0107$ \\ S-ALLYL-L-CYSTEINE ATTENUATES INFLAMMATION RELATED OXIDATIVE STRESS PARAMETERS AND INCREASES ADHESION CAPACITY OF PRIMARY HUMAN OSTEOARTHRITIC ARTICULAR CHONDROCYTES}

Zubeyir Elmazoglu ${ }^{1}$, Zehra Aydin Bek ${ }^{1}$, Berna Göker ${ }^{2}$, Berivan Bitik ${ }^{3}$, Cem Nuri Aktekin ${ }^{4}$, Mehmet Engin Tezcan ${ }^{5}$, Cimen Karasu ${ }^{1} .{ }^{1}$ Gazi University Faculty of Medicine, Cellular Stress Response and Signal Transduction Research Laboratory, Department of Medical Pharmacology, Ankara, Turkey, ${ }^{2}$ Gazi University Faculty of Medicine, Rheumatology, Ankara, Turkey; ${ }^{3}$ Baskent University, Rheumatology, Ankara, Turkey, ${ }^{4}$ Yıldırım Beyazıd University, Orthopedics andTraumatology, Ankara, Turkey; ${ }^{5}$ Kartal Dr. Lutfi Kirdar Training and Research Hospital, Rheumatology, Istanbul, Turkey

Background: Osteoarthritis $(O A)$ is characterized by progressive destruction of the articular cartilage, and chondrocytes, the only cells in articular cartilage, are in charge of maintaining the homeostasis of articular cartilage via modulating extracellular matrix anabolism and catabolism [1]. Due to the association of the degree of oxidative degeneration of chondrocytes and $O A$, preventing impaired redox signalling and oxidative death of chondrocyte are suggested as potential targets to relieve $O A[2]$. In this respect, some photochemicals have been shown to be potential agents for preventing or treating OA due to their antioxidant and antiinflammatory properties [3].

Objectives: We studied the effects of an antioxidant S-allyl-cysteine (SAC), a major sulphur-containing amino acid compound of garlic[4], on the redox system, and its associations with the proliferation rate and index of human OA chondrocytes(OACs).

Methods: Chondrocytes were isolated from the joint cartilages of OA patients (grade 4 , meanage $=66$ years, $B M l=29.7 \pm 4.4 \mathrm{~kg} / \mathrm{m}^{2}$ ). The alterations in cell proliferation (MTT), adhesion profile (RTCA-iCELLigence System), reactive oxygen species generation (ROS), lipid hydroperoxide levels (LPO), HNE-protein adduct levels (HNE), AGE-protein adduct levels(AGE), 3-nitrotyrosine levels (3-NT), 